\title{
HABITAT-QUALITY EFFECTS ON METAPOPULATION DYNAMICS IN GREATER WHITE-TOOTHED SHREWS, CROCIDURA RUSSULA
}

\author{
J. Jaquiéry, ${ }^{1}$ J. Guélat, T. Broquet, L. Berset-Brändli, E. Pelleggrini, R. Moresi, A. H. Hirzel, and N. Perrin \\ Department of Ecology and Evolution, University of Lausanne, CH-1015 Lausanne, Switzerland
}

\begin{abstract}
The effects of patch size and isolation on metapopulation dynamics have received wide empirical support and theoretical formalization. By contrast, the effects of patch quality seem largely underinvestigated, partly due to technical difficulties in properly assessing quality. Here we combine habitat-quality modeling with four years of demographic monitoring in a metapopulation of greater white-toothed shrews (Crocidura russula) to investigate the role of patch quality on metapopulation processes. Together, local patch quality and connectivity significantly enhanced local population sizes and occupancy rates $\left(R^{2}\right.$ $=14 \%$ and $19 \%$, respectively). Accounting for the quality of patches connected to the focal one and acting as potential sources improved slightly the model explanatory power for local population sizes, pointing to significant source-sink dynamics. Local habitat quality, in interaction with connectivity, also increased colonization rate $\left(R^{2}=28 \%\right)$, suggesting the ability of immigrants to target high-quality patches. Overall, patterns were best explained when assuming a mean dispersal distance of $800 \mathrm{~m}$, a realistic value for the species under study. Our results thus provide evidence that patch quality, in interaction with connectivity, may affect major demographic processes.
\end{abstract}

Key words: colonization; connectivity; Crocidura russula; ecological-niche factor analysis (ENFA); extinction; greater white-toothed shrew; habitat suitability; metapopulation dynamics; occupancy; Vallée de Joux, Switzerland.

\section{INTRODUCTION}

The concept of metapopulation refers to a set of local demes (or patches) connected by some level of migration and undergoing disequilibrium dynamics characterized by local extinctions and recolonizations (Hanski and Gaggiotti 2004). One of the earliest attempts to formalize these dynamics was Levins's (1969) patchoccupancy island model. Assuming that all local demes are equivalent in terms of extinction probability and connectivity, this model predicts expected occupancy rate as a function of extinction and colonization rates.

These first inroads prompted research on different fronts. On the empirical side, subsequent investigations have shown that Levins's simplifying assumptions of homogeneous rates are often not tenable. Local demes differ in extinction rate and connectivity, which affects metapopulation dynamics (e.g., Verboom et al. 1991, Kindvall and Ahlen 1992, Hanski and Thomas 1994, Eber and Brandl 1996). On the theoretical side, attempts were made to integrate these heterogeneities into metapopulation modeling (e.g., Hanski 1994, Hastings and Harrison 1994). Hanski and Ovaskainen (2000, 2003, see also Ovaskainen and Hanski 2001, 2004) contributed significantly to the theory of stochastic patch occupancy models by allowing patches to differ in size and connectivity. The condition for viability in a

Manuscript received 1 November 2007; accepted 31 January 2008. Corresponding Editor: F. S. Dobson.

${ }^{1}$ E-mail: julie.jaquiery@unil.ch given landscape depends on the "capacity" of the metapopulation, a function of the sizes and positions of its patches. The size of a patch (considered as a surrogate for local carrying capacity) affects its extinction rate and contribution to the migrant pool, while its isolation matters by affecting connectivity (i.e., contribution to recolonization).

Patch quality has rarely been explicitly considered in this context and might be seen as the main missing parameter in current metapopulation modeling (e.g., Thomas et al. 2001). Habitat quality is indeed bound to affect all processes determining metapopulation dynamics. On the one hand, high-quality patches may benefit from lower extinction rates (by enhancing individual survival or fecundity) or higher colonization rates (by attracting immigrants or facilitating settlement). On the other hand, they may send more migrants (owing to higher productivity), and thereby contribute more than others to recolonization processes. Patch quality might thus also provide an excellent surrogate for local carrying capacity. Although some studies have confirmed a role for habitat quality in occupancy, colonization, or extinction patterns (e.g., Thomas et al. 2001, Fleishman et al. 2002, Franken and Hik 2004), these topics remain largely underinvestigated, presumably due to practical difficulties in assessing habitat quality.

Recent developments in habitat-quality modeling are now tracing new inroads into this area. Habitat-quality modeling is emerging as a discipline of its own, building on a set of spatially explicit tools (geographic informa- 
tion systems) and associated statistics to identify environmental variables affecting a species' distribution and assess local habitat suitability (reviewed in Guisan and Zimmermann 2000). Up to now, applications to metapopulation studies have been mostly restricted to the delineation of local patches (e.g., Akçakaya et al. 1995, Akçakaya and Atwood 1997, Akçakaya 2000a, b). Efforts were made by Moilanen and Hanski (1998) to formally integrate habitat modeling into a metapopulation dynamics framework, but the predictive power of these models was not improved beyond the effects of patch area and isolation.

In the present paper, we combine spatially explicit modeling with a four-year demographic study to investigate the role of habitat quality on metapopulation dynamics in a small mammal, the greater white-toothed shrew (Crocidura russula). The species and study area (at the altitudinal margin of its distribution) were chosen on several grounds. First, owing to strong dependence on human habitations, local populations are discrete, highly structured, and easily identified. Second, owing to harsh climatic conditions, local populations are small and prone to local extinctions induced by demographic or environmental stochasticity. Third, this species displays high dispersal ability and short life span, which should combine with these features to induce high population turnover rates. Finally, its high trappability should facilitate mark and recapture studies. As metapopulation dynamics are poorly documented in mammals, this study system had the potential to contribute significantly to the field.

\section{Methods}

\section{Field sampling}

Crocidura russula is a small insectivorous mammal widespread in southwestern Europe. The life cycle is mostly annual (Jeanmaire-Besançon 1988) and the mating system mostly monogamous (Cantoni and Vogel 1989, Bouteiller and Perrin 2000). Breeding pairs defend territories where they may rear several litters from March to September (Jeanmaire-Besançon 1988). In the northern part of the species range (including the study area), individuals are preferentially anthropophilic below $600 \mathrm{~m}$ and strictly so at higher altitudes (Genoud 1985), relying on thermally favorable sites (farms, stables, and compost heaps) to meet the energetic needs of the cold season (Genoud and Hausser 1979). Local populations are thus linked to human habitations (villages or isolated farms), inducing a fragmented distribution (Fontanillas et al. 2004). The dispersal rate is high, mostly in female juveniles from the first litters (Favre et al. 1997).

The study area $(6 \times 14 \mathrm{~km})$ was located in the Vallee de Joux, Switzerland $\left(6^{\circ} 15^{\prime} \mathrm{E}, 46^{\circ} 37^{\prime} \mathrm{N}\right)$, an interior valley of the Swiss Jura at the altitudinal limit of the species range (1000-1300 $\mathrm{m}$ above sea level). The local populations are isolated from lowland populations by continuous mountain crests. Abundances were moni- tored in summer (August to September) in 106 sites, of which 62 were studied in 2003 and 84 in each of the years 2004, 2005, and 2006 (Fig. 1). Sampling sites consisted of $20 \times 20 \mathrm{~m}$ areas within private gardens. These were always adjacent to human habitations and usually comprised some lawn, a vegetable garden, a compost heap, a garden dwarf, wood piles, stone walls, and hedges. Forty-two of these sites were sampled over all four years, 40 sites over three consecutive years, two sites over two consecutive years, and 22 sites only once. Individuals were livetrapped using 20 Longworth smallmammal traps (B. N. Bolton, Inc, Vernon, British Columbia, Canada) per garden, baited with Tenebrio molitor larvae. After a pre-baiting period of two to four days, each site was visited four times during two consecutive days. The traps were opened daily around 06:30 hours, checked at 10:00 and at 13:30 hours, and then closed for the night. All caught individuals were individually marked by toe clipping and immediately released.

Local population size was estimated for each site as the mean number of individuals captured per year. A site occupancy rate was defined as the proportion of years in which at least one individual was captured. A site's colonization rate was estimated as the number of annual transitions from an empty to an occupied state, divided by the total number of transitions from an empty state. Extinction rate was similarly estimated as the number of transitions from an occupied to an empty state, divided by the total number of transitions from an occupied state.

\section{Habitat quality and connectivity}

Habitat quality (or suitability) was estimated using the ecological-niche factor analysis (ENFA; Biomapper 3.2; Hirzel et al. 2002, 2004) with the median algorithm (Hirzel and Arlettaz 2003) at a 25-m cell resolution. This multivariate analysis extracts a series of independent factors (linear combinations of environmental variables) that maximize the marginality and specialization of a focal species, relative to the reference area. Marginality measures how much environmental conditions in presence sites depart from average, and specialization measures the narrowness of their distributions, relative to that of reference sites. ENFA requires only presence data and is thus often applied when species absences do not necessarily reflect habitat unsuitability (e.g., Hirzel et al. 2001, Dettki et al. 2003, Reutter et al. 2003, Thomas 2003, Brotons et al. 2004, Engler et al. 2004, Chefaoui et al. 2005, Titeux et al. 2007). It is thus well adapted to our case, since absences may originate from stochastic demographic processes rather than environmental incompatibilities. The species was considered present on a site if at least one individual was captured over the four years.

Eight environmental variables were chosen in accordance with C. russula ecology (Hausser 1995; Table 1): three topographic variables (altitude, northness, and 


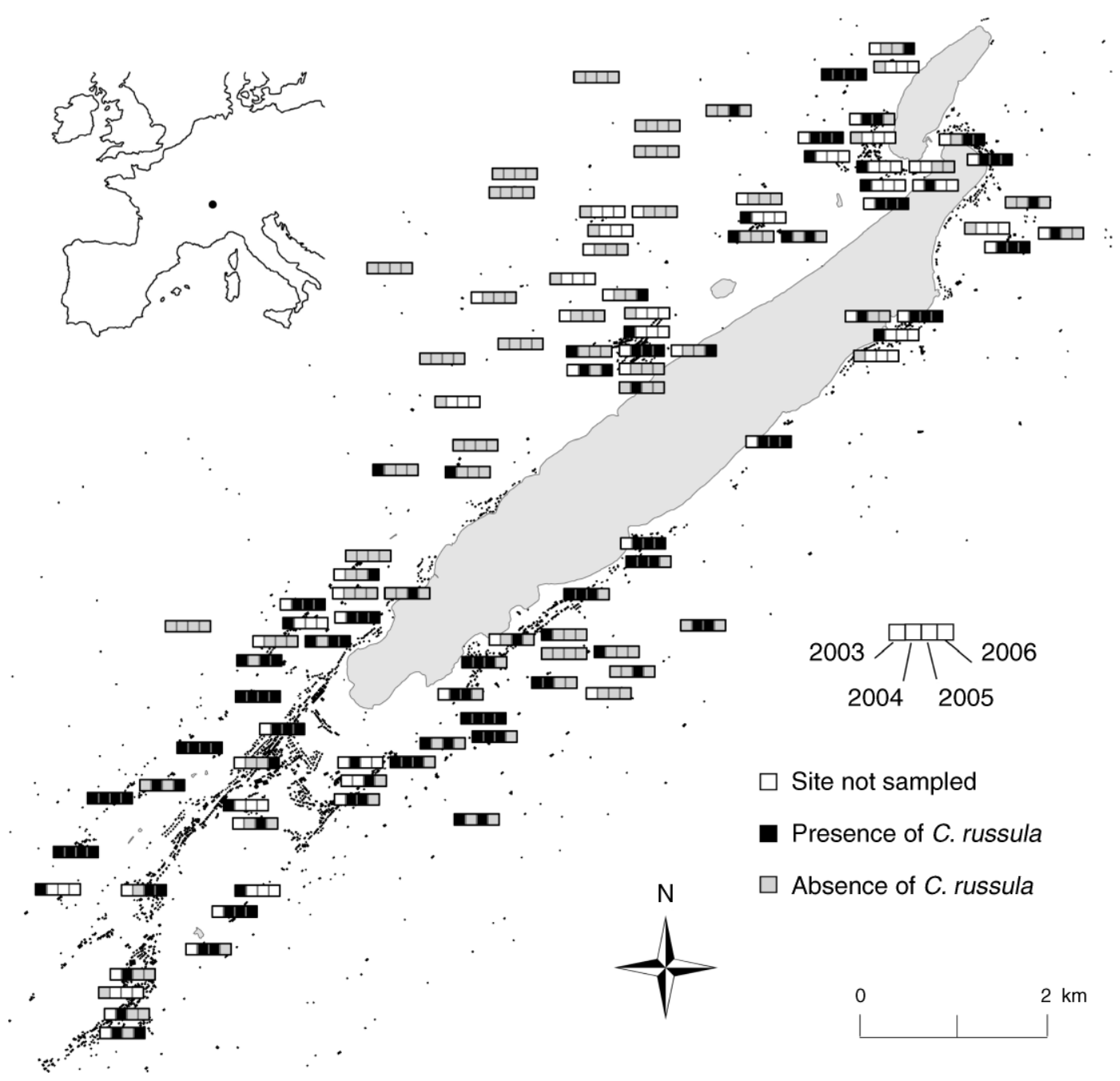

FIG. 1. Map of the study area (Vallée de Joux, Switzerland, with the Lac de Joux in gray). Each of the 106 rectangles represents one sampling site. The four squares within the rectangles indicate the occurrence pattern of the greater white-toothed shrew (Crocidura russula) for the four surveyed years (white square, not sampled; solid square, presence; gray square, absence).

eastness), two climatic variables (winter solar radiation and number of frost days per year), two anthropogenic variables (the number of winter-heated buildings within a $100 \mathrm{~m}$ radius and number of buildings within a $100 \mathrm{~m}$ radius weighted by distance), and one biotic variable measuring plant productivity (normalized difference vegetation index [NDVI]; Rouse et al. 1973). To reflect the sampling pattern, analysis was restricted to a $200-\mathrm{m}$ buffer around human habitations. The habitat quality (minimum 0, maximum 100) of any $25 \times 25 \mathrm{~m}$ cell within this area was calculated using the marginality factor plus all factors explaining more than $10 \%$ of specialization. The habitat quality of a site $\left(H_{i}\right)$ was then obtained as the mean over the $75 \times 75 \mathrm{~m}$ area surrounding the site (i.e., the focal cell plus its eight immediate neighbors).

All presence sites were used to calculate habitat quality for unoccupied or unsampled sites (full habitat model), but not for presence sites, in order to avoid any risk of circularity. Indeed, if the quality $H_{i}$ of a presence site $i$ were calculated from a set including itself, and that quality $H_{i}$ were used in turn to predict local occupancy or population size at site $i$ (which vary in part due to the contrast between presence and absence), then quality and demography might show some correlation by construction. Thus, when computing the quality of a presence site, we removed it, as well as its four neighboring sites (to get rid of any influence of possible connectivity effects) from the data set. A presence site will then be predicted as good only if it shares environmental features with other occupied sites (and not because it is itself part of the set chosen to define its own quality).

The quality and the robustness of the full habitat model was evaluated by the continuous Boyce index implemented in Biomapper 3.2 (Hirzel et al. 2006) using a $k$ fold cross-validation (Fielding and Bell 1997). The 
TABLE 1. Scores for environmental variables on the first four ENFA (ecological-niche factor analysis) axes for the greater whitetoothed shrew, Crocidura russula (full model including all 74 presence points).

\begin{tabular}{|c|c|c|c|c|c|c|}
\hline \multirow[b]{2}{*}{ Variable } & \multirow[b]{2}{*}{ Marginality } & \multicolumn{3}{|c|}{ Specialization factor } & \multicolumn{2}{|c|}{ Environmental variables (mean $\pm \mathrm{SD})$} \\
\hline & & 1 & 2 & 3 & Study area & Occupied sites \\
\hline BUILD $\dagger$ & 0.76 & 0.01 & -0.10 & 0.16 & $1 \pm 2.8$ & $8.8 \pm 6.7$ \\
\hline WHAB $\dagger$ & 0.56 & -0.17 & -0.11 & 0.24 & $1.2 \pm 3.4$ & $8.2 \pm 5.9$ \\
\hline NDVI & -0.24 & 0.33 & -0.34 & 0.55 & $0.32 \pm 0.11$ & $0.22 \pm 0.11$ \\
\hline ELEV† & -0.21 & -0.73 & 0.08 & 0.67 & $1159 \pm 132$ & $1055 \pm 57$ \\
\hline FROST & -0.05 & -0.18 & 0.02 & -0.38 & $17.3 \pm 4.3$ & $16.6 \pm 3.9$ \\
\hline EAST $\dagger$ & -0.01 & -0.02 & -0.32 & 0.07 & $-0.03 \pm 0.69$ & $-0.05 \pm 0.66$ \\
\hline NORTH§ & -0.01 & 0.23 & 0.14 & 0.05 & $0.05 \pm 0.72$ & $0.03 \pm 0.76$ \\
\hline WRAD $\S$ & 0.00 & 0.49 & 0.87 & 0.14 & $7099 \pm 1900$ & $7115 \pm 1435$ \\
\hline
\end{tabular}

Notes: Marginality measures how much environmental conditions in presence sites depart from average in the total area (a positive coefficient indicates that the species is found at values higher than average). Specialization factors measure the narrowness of the distribution of the environmental variables in presence sites relative to that of the total area (only absolute values matter for these axes; Hirzel et al. 2002). Environmental variables are: BUILD, number of habitations $(f)$ within a $100-\mathrm{m}$ radius, weighted by distance $(d)$ to the closest building, $f \exp [-(d / 100)]$; WHAB, number of buildings occupied in winter within a 100-m radius; NDVI, normalized difference vegetation index (correlated to vegetation biomass); ELEV, elevation; FROST, number of frost days per year; EAST, easterly aspect; NORTH, northerly aspect; WRAD, mean solar radiation in winter (December-February).

$\uparrow$ Data are available from the Federal Office of Topography, Wabern, Switzerland.

\$Data are available from $\langle$ http://asterweb.jpl.nasa.gov/index.asp $\rangle$.

$\S$ Data are available from the Swiss Federal Institute for Forest, Snow and Landscape Research, Birmensdorf, Switzerland.

presence data set was split into six partitions, then a model was built with five partitions (calibration data set) and validated with the omitted one (evaluation data set). We repeated this procedure for the six independent partitions to get the mean and the standard deviation of the Boyce index (Hastie et al. 2001).

A connectivity index $(S)$ was computed for each site $i$ as the sum of all potential sources weighted by distances, assuming a negative exponential kernel (Adler and Nürnberger 1994, Hanski 1994):

$$
S_{i}=\sum_{j \neq i}^{n} e^{-\alpha d_{i j}}
$$

where $n$ is the total number of sites (human habitations) in the study area $(n=2486), d_{i j}$ the distance from the focal patch $i$ to the source patch $j$, and $\alpha$ the factor weighting distance corresponding to the inverse of mean dispersal distance. As little is known on C. russula's mean dispersal distance, connectivity was evaluated for $1 / \alpha$ values ranging from $100 \mathrm{~m}$ to $5000 \mathrm{~m}$ (in $100-\mathrm{m}$ steps). A second connectivity index $\left(S_{H}\right)$, weighting connectivity by the habitat quality $\left(H_{j}\right)$ of the source patches, was similarly calculated as

$$
S_{H_{i}}=\sum_{j \neq i}^{n} e^{-\alpha d_{i j}} H_{j} .
$$

This second index differs from that used by Hanski (1994) in that patch quality replaces patch size as a surrogate for local carrying capacity. The additional explanatory power gained by considering habitat quality will be tested by comparing indices shown in Eqs. 1 and 2 .

\section{Regression models}

For each of the four response variables $y$ (namely mean population sizes, occupancy, colonization, and extinction rates), regression models were built as a function of local habitat quality $(H)$ and connectivity $(S)$ for a range of $\alpha$ values:

$$
y \sim H+S+\text { Interaction. }
$$

(Model 1)

A second series of models was then similarly built using the source-quality weighted connectivity index $\left(S_{H}\right)$ :

$$
y \sim H+S_{H}+\text { Interaction. }
$$

(Model 2)

We used weighted logistic regressions assuming a binomial distribution (or quasi-binomial if data were overdispersed), except for the mean population size where a linear regression was used (see Table 2). Significance levels were tested with an ANOVA ( $F$ distribution). The interaction term was removed when it remained nonsignificant for any $\alpha$ value.

Bootstrapping was used to test whether models accounting for habitat quality of source patches (model 2) explained significantly more (or less) variance in each of our response variables than model 1. For each $\alpha$ value, models 1 and 2 were fitted after bootstrapping the independent and response variables (with the same resampling vector for the two models), and the difference in explained variances (or deviances) between model 1 and model 2 was calculated. The procedure was repeated 1000 times, and the difference was considered significant if the $95 \%$ confidence interval did not include the 0 value (one-sided test). All analyses except ENFA were performed in R (R Development Core Team 2007).

\section{RESUlTS}

\section{Field sampling}

Approximately one-half of the sites were occupied each year (33 out of 62 in 2003, 40 out of 84 in 2004, 44 out of 84 in 2005, and 30 out of 84 in 2006; Fig. 1) with a 
TABLE 2. Regression models corresponding to the dispersal parameter $(\alpha)$ maximizing the amount of explained variance (or deviance).

\begin{tabular}{|c|c|c|c|c|c|c|c|c|c|c|c|c|c|}
\hline \multirow[b]{2}{*}{ Response variable } & \multirow[b]{2}{*}{ Final models } & \multirow[b]{2}{*}{$\alpha$} & \multirow[b]{2}{*}{$n$} & \multicolumn{3}{|c|}{$H$} & \multicolumn{3}{|c|}{$S$ or $S_{H}$} & \multicolumn{3}{|c|}{$I$} & \multirow{2}{*}{$\begin{array}{c}\text { Total } \\
R^{2}\end{array}$} \\
\hline & & & & Slope & $R^{2}$ & $P$ & Slope & $R^{2}$ & $P$ & Slope & $R^{2}$ & $P$ & \\
\hline \multirow[t]{2}{*}{ Mean population sizes $\dagger$} & $y \sim f(H+S)$ & $1 / 1000$ & 106 & + & 8.2 & $<0.01$ & + & 5.3 & $<0.05$ & & & & 13.5 \\
\hline & $y \sim f(H$ & & 106 & + & 8.2 & $<0.05$ & + & 6.2 & $<0.05$ & & & & 14.4 \\
\hline \multirow[t]{2}{*}{ Occupancy rates } & $y \sim f(H+S)$ & $1 / 800$ & 106 & + & 7.8 & $<0.001$ & + & 11.1 & $<0.001$ & & & & 18.9 \\
\hline & $y \sim f\left(H+S_{H}\right)$ & $1 / 800$ & 106 & + & 7.8 & $<0.001$ & + & 11.8 & $<0.001$ & & & & 19.6 \\
\hline \multirow[t]{2}{*}{ Colonization rates $\S$} & $y \sim f(H+S+I)$ & $1 / 800$ & 56 & - & 0.5 & 0.51 & - & 19.0 & $<0.001$ & + & 8.6 & $<0.01$ & 28.1 \\
\hline & $y \sim f\left(H+S_{H}+I\right)$ & $1 / 800$ & 56 & - & 0.5 & 0.51 & - & 19.3 & $<0.001$ & + & 8.5 & $<0.01$ & 28.3 \\
\hline \multirow[t]{2}{*}{ Extinction rates $\S$} & $y \sim f(H+S+I)$ & $1 / 500$ & 57 & - & 2.1 & 0.28 & - & 0.9 & 0.32 & + & 3.4 & 0.12 & 6.3 \\
\hline & $y \sim f(H$ & $1 / 300$ & 57 & - & 2.1 & 0.41 & - & 0.3 & 0.31 & + & 5.6 & 0.05 & 7.9 \\
\hline
\end{tabular}

Notes: Shown are the model retained, the regression type, the distribution used, the total amount of explained variance, and the number of sites $(n)$. For colonization models, $n$ represents the number of sites providing an opportunity to observe a colonization event (empty one year and monitored the following year). For extinction models, $n$ represents the number of sites providing an opportunity to observe an extinction event (occupied one year and monitored the following year). Also given for each variable are the sign of the slope, the explained variance, and the $P$ value for the explained variance $(H$, local habitat quality; $S$, connectivity; $S_{H}$, connectivity weighted by habitat quality of sources; $I$, interaction).

$\dagger$ Linear model with a Gaussian distribution.

\$ Weighted logistic model with a binomial distribution.

$\S$ Weighted logistic model with a quasi-binomial distribution.

total of 74 sites occupied at least once during the four years. Of the 84 sites sampled at least twice, 18 were occupied all years, 22 were never occupied, and 44 showed turnover. In the 62 sites that were both sampled at least twice and occupied at least once, we monitored 24 colonizations (over 51 possible events) and 40 extinctions (over 102 possible events).

Over the four years, 545 individuals were captured, which amounts to $3.8 \pm 3.3$ individuals $($ mean $\pm \mathrm{SD}$ ) per site occupied. The mean individual trapping probability, estimated as the number of captures per individual divided by the number of trapping sessions, was $0.47,0.45,0.40$, and 0.41 in 2003, 2004, 2005, and 2006 , respectively. Thus, the probability of missing one individual in four trapping sessions amounted to $\sim 10 \%$ $(0.08,0.09,0.13$, and 0.12 in 2003, 2004, 2005, and 2006, respectively) and the probability of missing both members of a breeding pair amounted to $\sim 1 \%(0.007$, $0.008,0.017$, and 0.014 in 2003, 2004, 2005, and 2006, respectively). No individual was recaptured over different sites or different years. Raw data are available in the online appendix of Guélat et al. (in press).

\section{Niche analysis}

The ENFA habitat model was built with four axes, explaining $100 \%$ of the marginality and $82 \%$ of the specialization. The environmental variable scores (Table 1) indicate a preference for high-density human settlements and winter-heated buildings. Solar radiation and altitude also played a role in niche specialization. The fit with actual occupation patterns was excellent, as quantified by a very high continuous Boyce index with a low variance $(0.88 \pm 0.07$; mean $\pm \mathrm{SD})$.

\section{Regression models}

Local population sizes and occupancy rates were positively affected by local habitat quality and connectivity. The regressions were significant for most dispersal values, with a peak in explained variance for a dispersal parameter $(1 / \alpha)$ of 1000 and $800 \mathrm{~m}$, respectively (model 1; dotted lines in Fig. 2). The model explanatory power increased slightly (significantly for local population sizes for dispersal values ranging 100-1300 m [gray area in Fig. 2] and marginally so for occupancy rate; $P=0.07$ for dispersal ranging 300-400 m) when accounting for the habitat quality of sources (model 2; solid lines in Fig. 2), with a peak in explained variance for a mean dispersal distance of $800 \mathrm{~m}$ in both response variables.

Colonization rate was significantly enhanced by the interaction between local habitat quality and connectivity for all dispersal values $>400 \mathrm{~m}$. Colonization actually increased with connectivity (Fig. 3), but more rapidly so for high-quality patches, hence the positive interaction. The explained variance also displayed a distinct peak for a mean dispersal value of $800 \mathrm{~m}$ (model 1; dotted line in Fig. 2), but was not significantly enhanced when accounting for the habitat quality of source patches (model 2; solid line in Fig. 2). By contrast, extinction rate remained essentially unaffected by habitat quality or connectivity, except for a very narrow range of dispersal values (200-300 m, model 2; solid line in Fig. 2), where a marginal effect stemmed from the interaction between local quality $(H)$ and connectivity weighted by source quality $\left(S_{H}\right)$.

Table 2 presents, for all models, the regression parameters corresponding to the dispersal values $(1 / \alpha)$ that best explain variances or deviances. The corresponding total proportion of variance explained by local habitat quality $(H)$, connectivity $(S)$, and interaction is low for extinction rates $\left(D^{2}=0.063\right)$, but moderate to high for the other response variables $\left(D^{2}=0.135\right.$ for mean population size, 0.189 for occupancy rates, and 0.281 for colonization rates). Accounting for the effect of source quality in the connectivity measure slightly increased the amount of explained variance (to 0.079, $0.144,0.196$, and 0.283 for extinction rate, population 


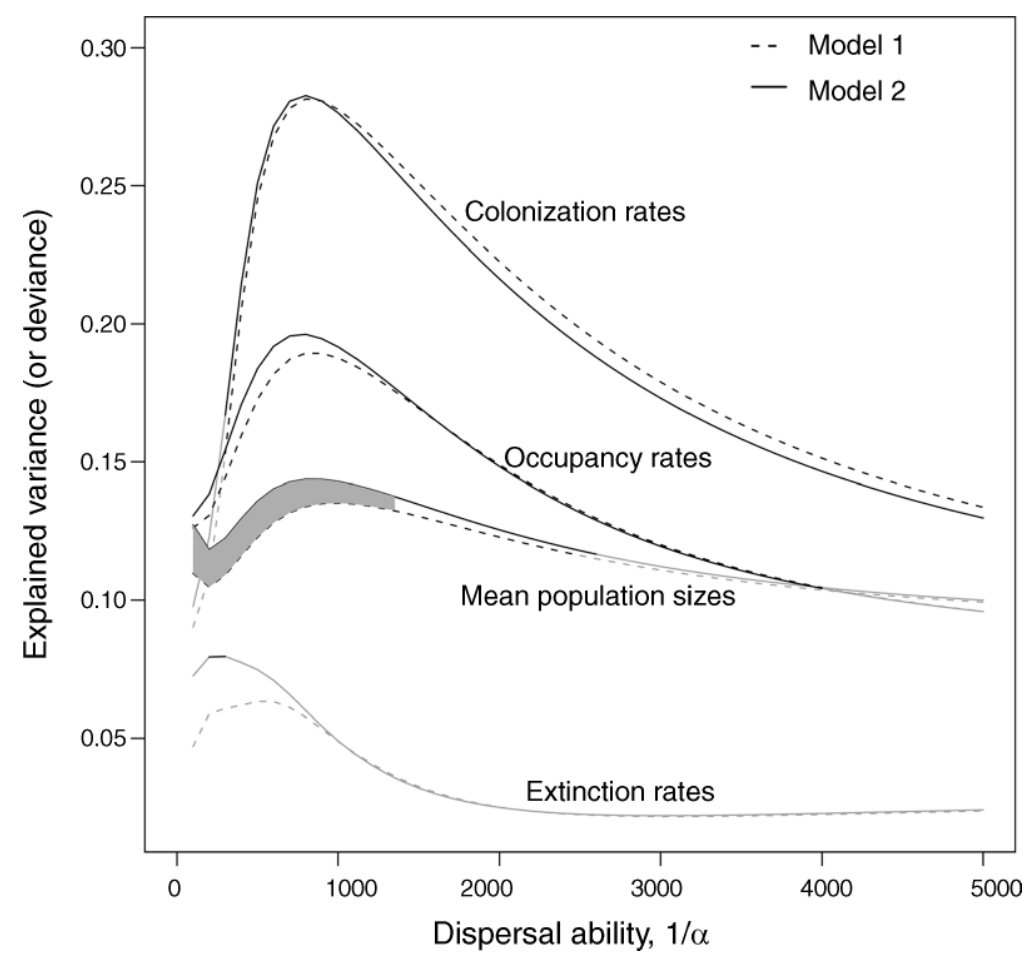

FIG. 2. Amount of explained variance (or deviance) as a function of the dispersal ability $(1 / \alpha)$ for all four response variables. Connectivity is weighted by the habitat quality of source populations in type 2 models (solid lines) but not in type 1 models (dashed lines). Interaction terms were removed from models for mean population sizes and occupancy rates but kept for models of colonization rate and extinction rate, being significant for some $\alpha$ values. Black lines (plain or dashed) highlight regions for which habitat quality had a significant effect on the response variable, and gray lines (plain or dashed) highlight regions for which habitat quality had no significant effect on the response variable. The gray area defines the region for which model 2 explained significantly more variance than model 1 .

sizes, occupancy rate, and colonization rate, respectively). Interestingly, for all three response variables clearly influenced by connectivity, the range of best-fit dispersal values is quite narrow, focusing on $800 \mathrm{~m}$ (Fig. 2).

\section{Discussion}

\section{Metapopulation dynamics}

The system under study clearly undergoes strong metapopulation dynamics, characterized by a high turnover rate. Local populations experienced an extinction or recolonization event every two to three years on average. Population sizes displayed a high variance, were poorly explained by environmental variables, and were typically quite small (about four individuals on average, which broadly corresponds to a single family). Demographic stochasticity thus certainly played a central role in their dynamics. But environmental stochasticity presumably was also important, given the localization of the metapopulation at the altitudinal margin of the species' distribution. Connectivity is bound to play a crucial role in this context, and dispersal must be important to counterbalance local extinctions. The significant influence of connectivity on local populations and occupancy rates points to important source-sink dynamics and rescue effects,

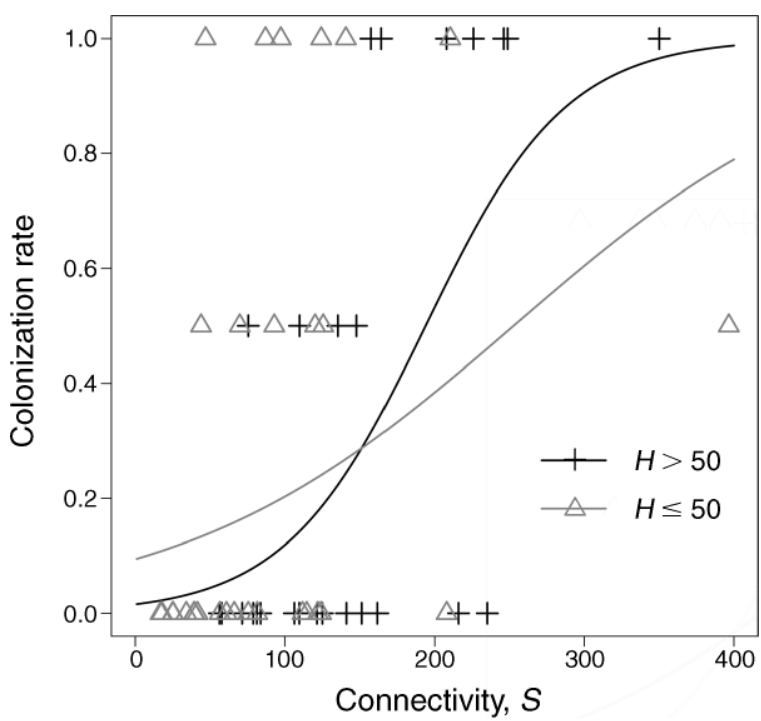

FIG. 3. Colonization rate increases with connectivity, but more rapidly for high-quality patches $(H>50$; black crosses $)$ than for low-quality patches $(H \leq 50$; gray triangles). Mean dispersal distance was fixed to $1 / \alpha=800 \mathrm{~m}$. 
whereby dispersers contribute markedly to local dynamics. Dispersal rate is known to be high in lowland populations, where about one-third of individuals are immigrants (Favre et al. 1997, Balloux et al. 1998, Bouteiller and Perrin 2000, Fontanillas et al. 2004). It might be still higher in highland populations, owing to the selective pressure set by metapopulation dynamics. Interestingly, our data provide an indirect estimate of dispersal distance. The three response variables that were clearly affected by connectivity (population size, occupancy rate, and colonization rate) displayed a distinct peak in explained variance for a mean dispersal value of $800 \mathrm{~m}$, a realistic value for a small mammal. This remains to be corroborated by direct mark and recapture experiments or by indirect inferences from genetic data.

\section{A role for habitat quality}

From our results, habitat quality significantly affected several main components of the metapopulation dynamics. Two effects must be disentangled here: the quality of the focal habitat and the quality of the source habitats that potentially send immigrants to the focal one.

Local habitat quality was shown to affect population size, occupancy rate, and colonization rate. The effect on population density was actually expected, presumably associated with an increased carrying capacity. Thomas et al. (2001), for instance, also showed that density correlates with habitat quality (measured as the percentage of feeding plants per patch weighted by species oviposition preferences) in several butterfly species. The effect on occupancy is more complex, since this rate results from a balance between extinction and colonization rates, both of which might a priori depend on local quality. Though our expectation of lower extinction rate in high-quality habitat (because of reduced environmental and demographic stochasticity) was not fulfilled, colonization rate did increase with local habitat quality. One might argue that this contrast between extinction and colonization could stem from the difference in the set of populations used in regressions: Only presence sites were used to test the effect of habitat quality on extinction (because extinctions can only occur in occupied sites), which should lower the variance in habitat quality and thereby the power to detect an effect. To test this, we also performed regressions of colonization rates on a data set restricted to presence sites (results not shown) and found the same qualitative results: Colonization rate still increased significantly with local habitat quality in interaction with connectivity. We conclude that this effect does not stem from the mere contrast between presence and absence sites (but mostly from the variance in quality among presence sites), and must result either from an active choice by dispersing individuals (implying the ability to target good-quality habitats) or by a higher probability of successful settlement in high-quality habitat. Thus, the effect of local quality on occupancy rate seems mediated mostly by colonization, a pattern similar to the one observed in a metapopulation of wolfspiders (Bonte et al. 2003), where local habitat quality was found to explain occupancy and colonization rates, but not extinction rate.

The crucial role of colonization was corroborated by the positive effects of connectivity on population size and occupancy rates. Our results suggest that a sizeable part of local populations consists of immigrants, who also affect occupancy rate through enhanced colonization and rescue effects (Brown and Kodric-Brown 1977). Local dynamics (driven by local fecundity and mortality rates) and regional dynamics (extinction and colonization events driven by emigration and immigration rates) apparently occur on similar time scales, a pattern characteristic of mass effects, defined as the quantitative effects of dispersal (both emigration and immigration) on local population dynamics (Leibold et al. 2004). In this context, the significant interaction between local habitat quality and connectivity on colonization rate (Fig. 3) is worth underlining: good patches are more easily colonized when they are well connected. Accounting for source-patch quality, however, did not improve the model explanatory power.

Our finding of a role for local habitat quality supports the findings of a few previous investigations on this topic. Local environmental variables, together with connectivity, increased the predictive power of metapopulation models in collared pikas (Franken and Hik 2004) and better explained occupancy and turnover patterns than did patch size and connectivity in the butterfly Speyeria nokomis apacheana (Fleishman et al. 2002).

By contrast, our finding of a role for habitat quality of source patches seems unprecedented. Moilanen and Hanski (1998) included such effects in their estimate of connectivity, but without improving predictive power. In the present instance, accounting for the quality of sources in connectivity measurements improved slightly, but significantly, the amount of variance explained in mean population sizes (and marginally so for occupancy rates). Interpretation seems straightforward: high-quality sources have a higher occupation probability and larger population sizes and, therefore, send more migrants. Sites well connected to high-quality sources should thus receive more migrants, and therefore display higher population sizes and occupancy rates.

It is worth underlining that, though significant, most effects detected here explain only a small fraction of the observed variance in demographic variables. This is certainly due in part to the strong stochasticity (both demographic and environmental) driving metapopulation dynamics in our study system, but possibly also to the effects of landscape features not included in our analysis, such as matrix quality or patch size. Matrix quality is expected to affect dispersal and connectivity in metapopulations (Ricketts 2001, Vandermeer et al. 2001, 
Haynes and Cronin 2003), and so might account for some of the residual variance in our study. However, documenting such effects would require information, not only on habitat quality and structure between patches, but also on patterns of individual dispersal, which is beyond the scope of the present study. Similarly, patch size, which is classically considered a key feature in metapopulation modeling, was not included here. Accounting for patch size (which does not a priori correlate with quality) may possibly explain some further residual variance, but the effect should be low, given the low variance in individual gardens. Size effects were further controlled by standardizing sampling sites $(20 \times 20 \mathrm{~m})$. Importantly, the power and interest of our approach is that quality can be readily derived from data banks of environmental variables (and thus easily estimated for all 2486 potential sites within our study area), which would obviously not be the case for garden sizes. One way to circumvent this problem might actually be to use habitat-quality modeling to define patch sizes (i.e., delimiting borders by a threshold-quality value; Akçakaya 2000a, b), an approach also beyond the scope of our present study.

Despite explaining a relatively limited part of total variance, the significant effects documented here provide compelling evidence that the quality of local patches, as well as that of potential sources, may affect several key demographic variables (including local population size, occupancy rate, and colonization rate), in some cases in interaction with connectivity. Besides their intrinsic interest, these results should encourage further efforts aimed at formally integrating habitat modeling with metapopulation dynamics. They also raise the possibility of using habitat suitability as a surrogate for occupation rate or population sizes. Allowing inclusion of nonsampled patches into connectivity measures and metapopulation dynamics should prove of significant practical importance, given that exhaustive sampling is often unfeasible. By the same token, this approach should also allow metapopulation investigations to be applied to more cryptic species than usually chosen in such studies.

\section{ACKNOWLEDGMENTS}

We benefited from discussions with Christophe Randin, Gwenaëlle Le Lay, and Jérôme Goudet on GIS and statistical analyses. Caroline Colliard, Antoine Burnier, and Sébastien Stücki provided help during the field sampling. Jon Yearsley and three anonymous referees made valuable comments on a previous draft of this manuscript. We thank N. E. Zimmermann and F. Kienast from the Swiss Federal Institute for Forest, Snow and Landscape Research, Birmensdorf, Switzerland. We are also grateful to the many "Combiers" who allowed us to trap in their private gardens. The Swiss National Science Foundation provided financial support (grant 3100A0108100 to N. Perrin).

\section{Literature Cited}

Adler, F. R., and B. Nürnberger. 1994. Persistence in patchy irregular landscapes. Theoretical Population Biology 45:4175 .
Akçakaya, H. R. 2000a. Conservation and management for multiple species: integrating field research and modeling into management decisions. Environmental Management 26:S75S83.

Akçakaya, H. R. 2000b. Viability analyses with habitat-based metapopulation models. Population Ecology 42:45-53.

Akçakaya, H. R., and J. L. Atwood. 1997. A habitat based metapopulation model of the California Gnatcatcher. Conservation Biology 11:422-434.

Akçakaya, H. R., M. A. McCarthy, and J. L. Pearce. 1995. Linking landscape data with population viability analysismanagement options for the helmeted honeyeater Lichenostomus melanops cassidix. Biological Conservation 73:169176.

Balloux, F., J. Goudet, and N. Perrin. 1998. Breeding system and genetic variance in the monogamous, semi-social shrew, Crocidura russula. Evolution 52:1230-1235.

Bonte, D., L. Lens, J. P. Maelfait, M. Hoffmann, and E. Kuijken. 2003. Patch quality and connectivity influence spatial dynamics in a dune wolfspider. Oecologia 135:227233.

Bouteiller, C., and N. Perrin. 2000. Individual reproductive success and effective population size in the greater whitetoothed shrew Crocidura russula. Proceedings of the Royal Society of London B 267:701-705.

Brotons, L., W. Thuiller, M. B. Araujo, and A. H. Hirzel. 2004. Presence-absence versus presence-only modelling methods for predicting bird habitat suitability. Ecography 27:437-448.

Brown, J. H., and A. Kodric-Brown. 1977. Turnover rates in insular biogeography - effect of immigration on extinction. Ecology 58:445-449.

Cantoni, D., and P. Vogel. 1989. Social organization and mating system of free-ranging, greater white-toothed shrews, Crocidura russula. Animal Behaviour 38:205-214.

Chefaoui, R. M., J. Hortal, and J. M. Lobo. 2005. Potential distribution modelling, niche characterization and conservation status assessment using GIS tools: a case study of Iberian Copris species. Biological Conservation 122:327-338.

Dettki, H., R. Lofstrand, and L. Edenius. 2003. Modeling habitat suitability for moose in coastal northern Sweden: empirical vs process-oriented approaches. AMBIO: A Journal of the Human Environment 32:549-556.

Eber, S., and R. Brandl. 1996. Metapopulation dynamics of the tephritid fly Urophora cardui: an evaluation of incidencefunction model assumptions with field data. Journal of Animal Ecology 65:621-630.

Engler, R., A. Guisan, and L. Rechsteiner. 2004. An improved approach for predicting the distribution of rare and endangered species from occurrence and pseudo-absence data. Journal of Applied Ecology 41:263-274.

Favre, L., F. Balloux, J. Goudet, and N. Perrin. 1997. Femalebiased dispersal in the monogamous mammal Crocidura russula: evidence from field data and microsatellite patterns. Proceedings of the Royal Society of London B 264:127-132.

Fielding, A. H., and J. F. Bell. 1997. A review of methods for the assessment of prediction errors in conservation presence/absence models. Environmental Conservation 24:38-49.

Fleishman, E., C. Ray, P. Sjogren-Gulve, C. L. Boggs, and D. D. Murphy. 2002. Assessing the roles of patch quality, area, and isolation in predicting metapopulation dynamics. Conservation Biology 16:706-716.

Fontanillas, P., E. Petit, and N. Perrin. 2004. Estimating sexspecific dispersal rates with autosomal markers in hierarchically structured populations. Evolution 58:886-894.

Franken, R. J., and D. S. Hik. 2004. Influence of habitat quality, patch size and connectivity on colonization and extinction dynamics of collared pikas Ochotona collaris. Journal of Animal Ecology 73:889-896.

Genoud, M. 1985. Ecological energetics of two European shrews-Crocidura russula and Sorex coronatus (Soricidae, Mammalia). Journal of Zoology 207:63-85. 
Genoud, M., and J. Hausser. 1979. Ecology of a Crocidura russula population in a rural mountain habitat. Terre et Vie 33:539-554.

Guélat, J., J. Jaquiéry, L. Berset-Brändli, E. Pellegrini, R. Moresi, T. Broquet, A. H. Hirzel, and N. Perrin. 2008. Mass effects mediate coexistence in competing shrews. Ecology 89, in press.

Guisan, A., and N. E. Zimmermann. 2000. Predictive habitat distribution models in ecology. Ecological Modelling 135: 147-186.

Hanski, I. 1994. A practical model of metapopulation dynamics. Journal of Animal Ecology 63:151-162.

Hanski, I., and O. E. Gaggiotti. 2004. Ecology, genetics and evolution of metapopulations. Elsevier Academic Press, London, UK.

Hanski, I., and O. Ovaskainen. 2000. The metapopulation capacity of a fragmented landscape. Nature 404:755-758.

Hanski, K., and O. Ovaskainen. 2003. Metapopulation theory for fragmented landscapes. Theoretical Population Biology 64:119-127.

Hanski, I., and C. D. Thomas. 1994. Metapopulation dynamics and conservation - a spatially explicit model applied to butterflies. Biological Conservation 68:167-180.

Hastie, T., R. Tibshirani, and J. H. Friedman. 2001. The elements of statistical learning: data mining, inference, and prediction. Springer-Verlag, New York, New York, USA

Hastings, A., and S. Harrison. 1994. Metapopulation dynamics and genetics. Annual Review of Ecology and Systematics 25: $167-188$.

Hausser, J. 1995. Mammifères de la Suisse. Commission des Mémoires de l'Académie Suisse des Sciences Naturelles, Basel, Switzerland.

Haynes, K. J., and J. T. Cronin. 2003. Matrix composition affects the spatial ecology of a prairie planthopper. Ecology 84:2856-2866.

Hirzel, A. H., and R. Arlettaz. 2003. Modeling habitat suitability for complex species distributions by environmental-distance geometric mean. Environmental Management 32:614-623

Hirzel, A. H., J. Hausser, D. Chessel, and N. Perrin. 2002. Ecological-niche factor analysis: how to compute habitatsuitability maps without absence data? Ecology 83:20272036.

Hirzel, A. H., J. Hausser, and N. Perrin. 2004. Biomapper 3.1. Statistical software. University of Lausanne, Laboratory for Conservation Biology, Lausanne, Switzerland. 〈http://www2. unil.ch/biomapper/ $>$

Hirzel, A. H., V. Helfer, and F. Métral. 2001. Assessing habitatsuitability models with a virtual species. Ecological Modelling 145:111-121.

Hirzel, A. H., G. Le Lay, V. Helfer, C. Randin, and A. Guisan. 2006. Evaluating the ability of habitat suitability models to predict species presences. Ecological Modelling 199:142-152.

Jeanmaire-Besançon, F. 1988. Sexual maturity of Crocidura russula. Acta Theriologica 33:477-485.
Kindvall, O., and I. Ahlen. 1992. Geometrical factors and metapopulation dynamics of the bush cricket, Metrioptera bicolor philippi (Orthoptera, Tettigoniidae). Conservation Biology 6:520-529.

Leibold, M. A., M. Holyoak, N. Mouquet, P. Amarasekare, J. M. Chase, M. F. Hoopes, R. D. Holt, J. B. Shurin, R. Law, D. Tilman, M. Loreau, and A. Gonzalez. 2004. The metacommunity concept: a framework for multi-scale community ecology. Ecology Letters 7:601-613.

Levins, R. 1969. Some demographic and genetic consequences of environmental heterogeneity for biological control Bulletin of Entomological Society of America 15:237-240.

Moilanen, A., and I. Hanski. 1998. Metapopulation dynamics: effects of habitat quality and landscape structure. Ecology 79:2503-2515.

Ovaskainen, O., and I. Hanski. 2001. Spatially structured metapopulation models: global and local assessment of metapopulation capacity. Theoretical Population Biology 60:281-302.

Ovaskainen, O., and I. Hanski. 2004. Metapopulation dynamics in highly fragmented landscapes. Pages 73-104 in I. Hanski and O. E. Gaggiotti, editors. Ecology, genetics and evolution of metapopulations. Elsevier Academic Press, Amsterdam, The Netherlands.

R Development Core Team. 2007. R: a language and environment for statistical computing. R Foundation for Statistical Computing. Vienna, Austria.

Reutter, B. A., V. Helfer, A. H. Hirzel, and P. Vogel. 2003. Modelling habitat-suitability on the base of museum collections: an example with three sympatric Apodemus species from the Alps. Journal of Biogeography 30:581-590.

Ricketts, T. H. 2001. The matrix matters: effective isolation in fragmented landscapes. American Naturalist 158:87-99.

Rouse, J. W., R. H. Haas, J. A. Schell, and D. W. Deering. 1973. Monitoring vegetation systems in the great plains with ERTS. Third ERTS Symposium:309-317.

Thomas, C. J. 2003. Anopheles gambiae and climate in Brazil. Lancet Infectious Diseases 3:326-326.

Thomas, J. A., N. A. D. Bourn, R. T. Clarke, K. E. Stewart, D. J. Simcox, G. S. Pearman, R. Curtis, and B. Goodger. 2001. The quality and isolation of habitat patches both determine where butterflies persist in fragmented landscapes. Proceedings of the Royal Society of London B 268:17911796.

Titeux, N., M. Dufrene, J. Radoux, A. H. Hirzel, and P. Defourny. 2007. Fitness-related parameters improve presence-only distribution modelling for conservation practice: the case of the Red-backed Shrike. Biological Conservation 138:207-223.

Vandermeer, J., and R. Carvajal. 2001. Metapopulation dynamics and the quality of the matrix. American Naturalist 158:211-220.

Verboom, J., A. Schotman, P. Opdam, and J. A. J. Metz. 1991. European nuthatch metapopulations in a fragmented agricultural landscape. Oikos 61:149-156. 